\title{
A polarimetric scattering database for non-spherical ice particles at microwave wavelengths
}

\author{
Yinghui Lu ${ }^{1,2, a}$, Zhiyuan Jiang ${ }^{1}$, Kultegin Aydin ${ }^{2}$, Johannes Verlinde ${ }^{1}$, Eugene E. Clothiaux ${ }^{1}$, and Giovanni Botta ${ }^{1,2, b}$ \\ ${ }^{1}$ The Dept. of Meteorology and Atmospheric Science, The Pennsylvania State University, \\ University Park, Pennsylvania, USA \\ ${ }^{2}$ The Dept. of Electrical Engineering, The Pennsylvania State University, University Park, \\ Pennsylvania, USA \\ anow at: Climate and Ecosystem Sciences Division, Lawrence Berkeley National Laboratory, \\ Berkeley, California, USA \\ b now at: Google, New York, USA
}

Correspondence to: Kultegin Aydin (aydin@engr.psu.edu)

Received: 6 July 2016 - Published in Atmos. Meas. Tech. Discuss.: 13 July 2016

Revised: 24 September 2016 - Accepted: 26 September 2016 - Published: 19 October 2016

\begin{abstract}
The atmospheric science community has entered a period in which electromagnetic scattering properties at microwave frequencies of realistically constructed ice particles are necessary for making progress on a number of fronts. One front includes retrieval of ice-particle properties and signatures from ground-based, airborne, and satellite-based radar and radiometer observations. Another front is evaluation of model microphysics by application of forward operators to their outputs and comparison to observations during case study periods. Yet a third front is data assimilation, where again forward operators are applied to databases of ice-particle scattering properties and the results compared to observations, with their differences leading to corrections of the model state.

Over the past decade investigators have developed databases of ice-particle scattering properties at microwave frequencies and made them openly available. Motivated by and complementing these earlier efforts, a database containing polarimetric single-scattering properties of various types of ice particles at millimeter to centimeter wavelengths is presented. While the database presented here contains only single-scattering properties of ice particles in a fixed orientation, ice-particle scattering properties are computed for many different directions of the radiation incident on them. These results are useful for understanding the dependence of iceparticle scattering properties on ice-particle orientation with respect to the incident radiation. For ice particles that are
\end{abstract}

small compared to the wavelength, the number of incident directions of the radiation is sufficient to compute reasonable estimates of their (randomly) orientation-averaged scattering properties.

This database is complementary to earlier ones in that it contains complete (polarimetric) scattering property information for each ice particle - 44 plates, 30 columns, 405 branched planar crystals, 660 aggregates, and 640 conical graupel - and direction of incident radiation but is limited to four frequencies (X-, Ku-, Ka-, and W-bands), does not include temperature dependencies of the single-scattering properties, and does not include scattering properties averaged over randomly oriented ice particles. Rules for constructing the morphologies of ice particles from one database to the next often differ; consequently, analyses that incorporate all of the different databases will contain the most variability, while illuminating important differences between them. Publication of this database is in support of future analyses of this nature and comes with the hope that doing so helps contribute to the development of a database standard for ice-particle scattering properties, like the NetCDF (Network Common Data Form) CF (Climate and Forecast) or NetCDF CF/Radial metadata conventions. 


\section{Introduction}

Cloud and precipitation processes are important components of the climate system of Earth and have been gaining increased attention in recent years. The importance of clouds and precipitation to climate and the uncertainties clouds introduce into future climate projections have emerged as such important topics that a new chapter in the most recent assessment of the Intergovernmental Panel on Climate Change (IPCC) is now dedicated to clouds and aerosols (IPCC, 2013; Chapter 7). As this chapter makes clear, amongst the many outstanding issues related to clouds and precipitation are those that result from ice-particle properties and processes, issues that must be solved to reduce uncertainties related to clouds and precipitation in future climate assessments.

Passive and active microwave-based observations of cloud- and precipitating-ice processes have an important role to play in improving our understanding of them and their impacts. This includes, for example, observational characterization of cloud and precipitation properties via the core Global Precipitation Measurement (GPM) mission (e.g., Hou et al., 2014), assimilation of cloud- and precipitation-affected microwave radiances in operational numerical weather prediction schemes (e.g., Geer and Baordo, 2014), and assessment of cloud-ice microphysics parameterization performance (e.g., Galligani et al., 2015). As studies on these topics have advanced, so has awareness of the importance of developing models of ice particles with realistic shapes (e.g., Westbrook et al., 2004; Maruyama and Fujiyoshi 2005) and radiative scattering properties (e.g., Kim, 2006; Liu, 2008; Petty and Huang, 2010; Kulie et al., 2010; Johnson et al., 2012). As ice-particle research has gained momentum, so has the importance of ice-particle identification via remote sensing, leading to many studies on the value of multiple frequencies (e.g., Matrosov, 1998; Kneifel et al., 2011, 2015; Leinonen et al., 2012, 2015; Battaglia et al., 2014; Kulie et al., 2014; Stein et al., 2015; Leinonen and Moisseev, 2015), polarization (e.g., Straka et al., 2000; Aydin and Singh, 2004; Chandrasekar et al., 2013; Kumjian, 2013), and the two combined (e.g., Tyynelä and Chandrasekhar, 2014) in their identification. Polarimetric, multi-frequency measurements also provide one path forward for quantitative estimation of ice water content (IWC). For example, Aydin and Tang (1997), using 94 and $220 \mathrm{GHz}$ radar frequencies; Ryzhkov et al. (1998), using a $3 \mathrm{GHz}$ radar frequency; and $\mathrm{Lu}$ et al. (2015), using multiple radar frequencies, derived relationships between IWC and polarimetric observables such as $K_{\mathrm{DP}}$ and $Z_{\mathrm{DR}}$.

To develop ice-particle retrieval algorithms and forward radiative operators, one must know the single-scattering properties (e.g., backscattering cross section) of the ice particles in clouds and precipitation, which are quite challenging to calculate because of their complex morphologies. Examples of numerical methods capable of calculating single- scattering properties of ice particles with arbitrary shapes are the discrete dipole approximation (DDA; Purcell and Pennypacker, 1973; Draine and Flatau, 1994; Yurkin and Hoekstra, 2011), the finite-difference time domain method (FDTD; Yee, 1966; Tavlove and Hagness, 2005), the pseudo-spectral time domain method (Liu et al., 2012), the generalized multiparticle Mie method (GMM; Xu, 1995), and the recently developed invariant embedding (Bi and Yang, 2014) and superposition (Mackowski and Mishchenko, 1996; Mackowski, 2014) T-matrix methods. Though accurate, these methods are often computationally expensive and are not yet practical in running online calculations. Instead, pre-calculated databases of ice-particle scattering properties at microwave wavelengths are created and made publicly available, such as those published by Kim (2006), Liu (2008), Nowell et al. (2013), Tyynelä and Chandrasekar (2014), and Kuo et al. (2016) at microwave wavelengths. However, these existing databases use randomly oriented ice particles, for which some polarimetric information is lost, such as differential reflectivity $\left(Z_{\mathrm{DR}}\right)$ and specific differential phase $\left(K_{\mathrm{DP}}\right)$, and they are thus not suitable for all types of polarimetric studies.

In this work scattering calculations for ice aggregates (Botta et al., 2010, 2011), branched planar ice crystals (Botta et al., 2013; referred to as "dendrites" in their paper), ice plates and columns ( $\mathrm{Lu}$ et al., 2015), and conical graupel (Oue et al., 2015) are assembled and synthesized into one self-consistent set of scattering properties. The results are arranged into a database containing single-scattering properties of ice particles at frequencies of $9.4 \mathrm{GHz}$ (X-band), $13.4 \mathrm{GHz}$ (Ku-band), $35.6 \mathrm{GHz}$ (Kaband), and $94.0 \mathrm{GHz}$ (W-band) and for different orientations of the ice particles relative to the incident radiation, which permits their use in all polarimetric studies. The database is now publically available for interested investigators. The remaining parts of this paper describe the particle morphologies and the numerical methods used in calculating the ice-particle single-scattering properties (Sect. 2), the scattering geometry (Sect. 3), the scattering properties calculated (Sect. 4), the structure of the database (Sect. 5), and some illuminative results from the database (Sect. 6), followed by a brief summary and description of future refinements to the database (Sect. 7).

\section{Calculating single-scattering properties of individual ice particles}

The numerical methods we used to create the database are the GMM method implemented by Xu (1995) and the DDA method implemented by Yurkin and Hoekstra (2011). Both of these numerical methods have the flexibility to model ice particles with arbitrary shapes. When using the GMM method, which calculates scattering properties of clusters of non-overlapping spheres, the ice particles are modeled using 
Table 1. Dielectric constants at $0^{\circ} \mathrm{C}$ for $\mathrm{W}$-, $\mathrm{Ka}$-, $\mathrm{Ku}$-, and $\mathrm{X}$-band wavelengths of pure ice and of the tiny spheres used in the representations of the plates, branched planar crystals, and columns after compensating for the air gaps.

\begin{tabular}{llrrrr}
\hline \multicolumn{2}{c}{ Band (wavelength in mm) } & $\mathrm{W}(3.19)$ & $\mathrm{Ka}(8.40)$ & $\mathrm{Ku}(22.4)$ & $\mathrm{X}(31.86)$ \\
\hline \multirow{2}{*}{ Pure ice } & Real part & 3.1682 & 3.1683 & 3.1686 & 3.1688 \\
& Imaginary part $\left(10^{-4}\right)$ & 3.2586 & 6.5053 & 13.044 & 16.777 \\
\hline Branched planar crystal/ & Real part & 5.6531 & 5.6536 & 5.6546 & 5.6552 \\
plate adjusted (69\%) & Imaginary part $\left(10^{-4}\right)$ & 10.356 & 20.676 & 41.462 & 53.332 \\
\hline \multirow{2}{*}{ Column adjusted (74\%) } & Real part & 4.9272 & 4.9275 & 4.9283 & 4.9288 \\
& Imaginary part $\left(10^{-4}\right)$ & 7.911 & 15.794 & 31.672 & 40.738 \\
\hline
\end{tabular}

clusters of closely packed tiny (orders of magnitude smaller than the wavelength) spheres that collectively resemble the shape of the ice particles. Modeling ice aggregates using this approach is convenient due to its flexibility in constructing arbitrary shapes using clusters of spheres (Botta et al., 2010, 2011).

The DDA method approximates an ice particle as a cluster of polarizable points. This method calculates scattering properties of the cluster by considering both the dipoles induced by the incident electric field at these polarizable points and the interactions between these dipoles. Although not required, the polarizable points are often arranged in cubic lattices to accelerate calculations using the fast Fourier transform (Goodman et al., 1991); thus the DDA method is suitable for calculating scattering properties of compact particles such as ice columns and plates. A cubic lattice arrangement for the polarizable points is less efficient computationally for sparse particles because of the many gaps within the lattice (Petty and Huang, 2010). As such, we do not use the DDA method to calculate scattering properties of ice aggregates.

Although the GMM and DDA methods provide scattering results with accuracies specified in the calculations, they do not provide identical results because they model ice particles in different ways (i.e., GMM as clusters of spheres and DDA as dipoles). However, the shape differences between the two representations of the same ice particle do not appear critical for ice-particle scattering studies because of the variability in ice-particle morphologies. Differences in the representation of a conical ice-particle shape are overwhelmed by the differences of any conical shape with respect to similarly shaped (but not identical) particles in nature. The morphologies of the ice particles in the database and the methods used to represent them in GMM and DDA calculations are now summarized.

\subsection{Aggregates}

The detailed algorithm for generating ice aggregates is described in Botta et al. (2010, 2011). Only the GMM method was used for ice-aggregate scattering calculations. Ice aggregates were constructed using ice columns (modeled as a line of spheres) and stellar ice crystals (modeled as three identical lines of spheres sharing a common central sphere). The dimensional relationship used for the columns was

$d=3.527 \times 10^{-2} L^{0.437}$

where $d$ and $L$ are, respectively, the width and length of the columns (in centimeters) (Pruppacher and Klett, 1997, p. 51, Table 2.2b, N1e). The dimensional relationship used for the stellar crystals was

$h=9.96 \times 10^{-3} d^{0.415}$,

where $h$ and $d$ are, respectively, the thickness and maximum dimension of the stellar crystal (in centimeters) (Pruppacher and Klett, 1997, p. 51, Table 2.2a, P1d). The dielectric constant of the spheres was set to that of pure ice at $0^{\circ} \mathrm{C}$ (See Table 1).

The sizes and shapes of the ice aggregates were characterized by their maximum dimensions (i.e., the maximum horizontal dimension of the cluster) and their aspect ratios (i.e., the ratio between the maximum vertical and horizontal dimensions). The ice-aggregate generation algorithm created each ice-aggregate realization by first specifying a reference spheroid whose maximum horizontal dimension and aspect ratio determined those of the ice-aggregate realization. Then a column or stellar crystal monomer was added to the aggregate one at a time, and the parts of the column or stellar crystal that were outside of the reference spheroid were removed. This procedure was repeated until the aggregate reached the desired mass within the specified tolerance. Two mass-dimensional relationships (P1d and P1c in Mitchell, 1996) were used to represent low-density (LD) and highdensity (HD) aggregates. For LDt-P1d aggregates in Table 2, the connecting point between the current aggregate and the newly added stellar is randomly selected among the locations at the tips, while for the remaining types of aggregates the connecting point is randomly selected among all locations. For the ice aggregates in the database the aspect ratios of the reference spheroids were set to 0.6. (The maximum horizontal dimensions of the reference spheroids are listed in $\mathrm{Ta}-$ ble 2.) However, the maximum dimensions and aspect ratios 
Table 2. Species and size information for the ice particles in the database. The number within parentheses below each ice-particle species is the total number of ice particles of that species in the database. The numbers in parentheses following the maximum dimensions of the aggregates (maximum dimensions of the reference spheroids for the aggregates), branched planar crystals, plates, and columns indicate the number of realizations (i.e., different ice particles) with that size. For conical graupel, with one realization for each combination of density, cone angle, and equal-volume-sphere radius, there are a total of 640 graupel particles in the database.

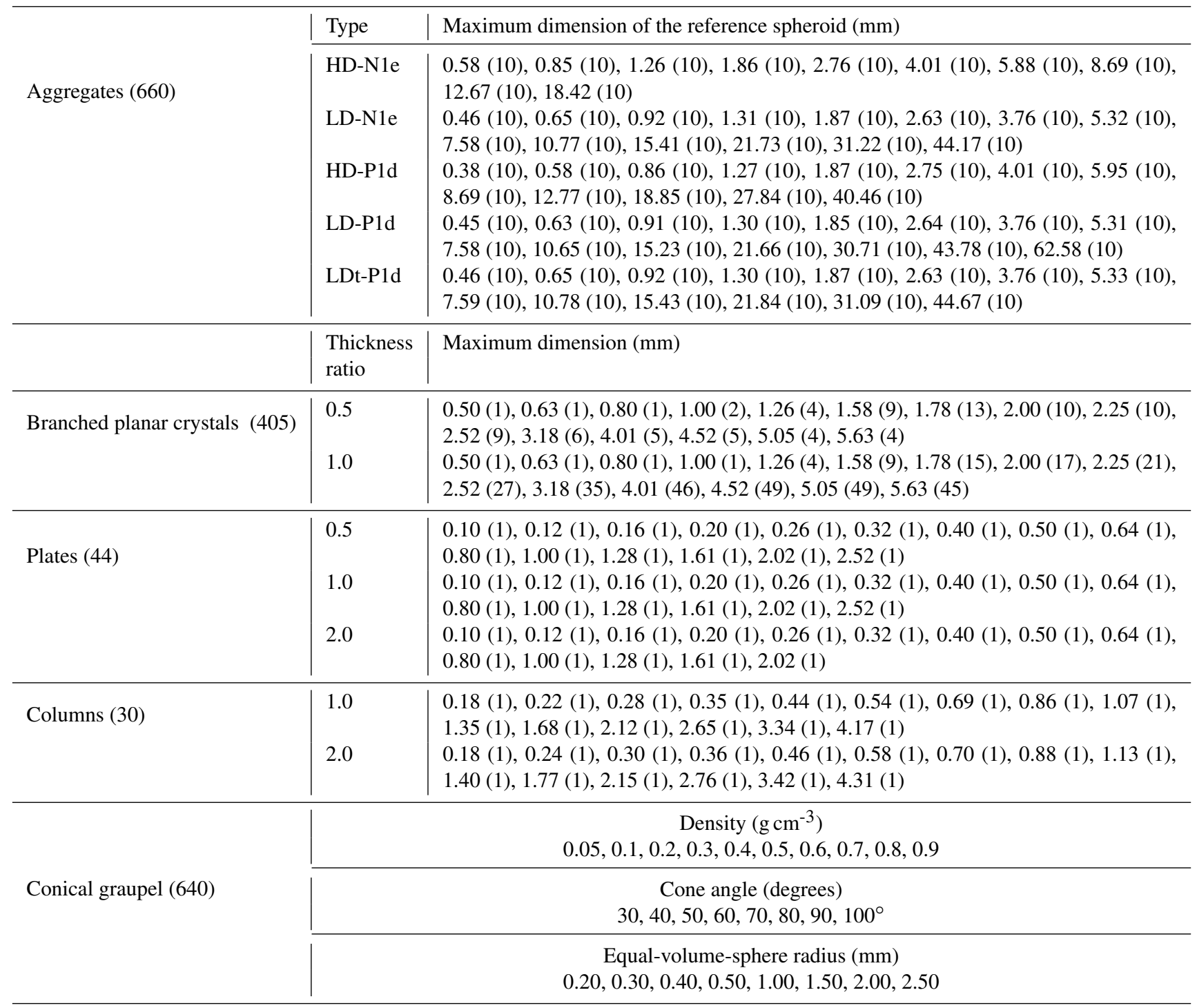

of the aggregates generated in this manner were not necessarily those of the circumscribing spheroids. For example, to build large aggregates with small amounts of mass, large monomers have to be used, and the resulting aggregates are flat (Botta et al., 2011).

\subsection{Branched planar crystals and plates}

The morphologies of branched planar crystals in nature are characterized by several features: their hexagonal core size (if present), branch widths, sub-branch widths and locations, number of sub-branches, and spacing between subbranches. For the purpose of capturing different ice-crystal morphologies, the properties of these different features must be varied to obtain different realizations of branched planar crystals.

Botta et al. (2013, their appendix A) describe the representations of branched planar crystals used in the GMM calculations. To construct the database, the maximum dimensions of the branched planar crystals in the database ranged from 0.50 to $5.63 \mathrm{~mm}$, equally spaced in logarithmic space. The thickness of a branched planar crystal as a function of maximum dimension was given by the dimensional relationship

$h=9.022 \times 10^{-3} d^{0.377}$, 
where $h$ and $d$ are the thickness and maximum dimension of the branched planar crystal (in centimeters) (Pruppacher and Klett, 1997, p. 51, Table 2.2b, P1e). To increase the variability of the branched planar crystals in the database, a second thickness of the branched planar crystals was obtained by multiplying the thickness $h$ above by 0.5 ; therefore, each branched planar crystal had one of two reference thickness variation factors: 1.0 (the default thickness) and 0.5 (the halved thickness). There were 405 different realizations of branched planar crystals whose single-scattering properties were computed and are now included in the database.

For plates, i.e., planar crystals with no branches, maximum dimensions ranged from 0.10 to $2.52 \mathrm{~mm}$, equally spaced in logarithmic space. The thicknesses of the plates were given by the dimensional relationship

$h=1.41 \times 10^{-2} d^{0.474}$,

where $h$ and $d$ are the thickness and maximum dimension of the plates (in centimeters) (Pruppacher and Klett, 1997, p. 51, Table 2.2a, P1a). In the case of plates, thickness variation factors of $0.5,1.0$, and 2.0 were used to increase the range of variability of their properties. A total of 44 realizations of plates are now included in the database.

Representations of branched planar crystals and plates for GMM calculations were as three layers of tiny spheres closely packed in the face-centered cubic lattice (FCC), which achieves a maximum packing factor of $74 \%$ for an infinite lattice (Botta et al., 2013; Lu et al., 2013, 2014b). Thus the radius of the tiny spheres was determined by the thickness of the planar ice crystals. For branched planar crystals with the same maximum dimensions, the crystals with half of the reference thickness needed significantly larger numbers of tiny spheres to model than the ones with the reference thickness. For some of the large, thin planar ice crystals with broad branches, the numbers of tiny spheres used to represent them exceeded the capability of the GMM code within our computational framework, and these realizations of planar ice crystals were discarded. Because the largest planar crystals tend to grow as open-structure crystals with thin branches, as opposed to broad branches (Takahashi et al., 1991), the lack of this planar ice crystal type from the database may not be detrimental.

In Botta et al. (2013) and Lu et al. (2013) pure ice dielectric constants at $0^{\circ} \mathrm{C}$ were used for the tiny spheres in the GMM representations of planar ice crystals. However, when considering the gaps between the tiny spheres in the GMM representations of planar ice crystals, the ice crystals were mixtures of the tiny spheres and the air gaps between them. Because of this mixture of tiny spheres of pure ice and air, the dielectric constant of the ice crystal (the "mixture") was smaller than that of the pure ice in the planar crystal. In order to match the effective dielectric constant of the GMM representation of an ice crystal to that of pure ice at $0^{\circ} \mathrm{C}$, the dielectric constants of the tiny spheres in this representation were adjusted according to Lu et al. (2014a). In this approach the effective dielectric constant of the mixture of tiny spheres and air gaps in the GMM representation of an ice crystal was set to the dielectric constant of pure ice at $0^{\circ} \mathrm{C}$. Then the Maxwell-Garnett equation was inverted to solve for the dielectric constant of the tiny spheres necessary to achieve it:

$\epsilon_{\text {spheres }}=\epsilon_{\text {air }}\left[1+3 /\left(f\left(\epsilon_{\text {ice }}+2 \epsilon_{\text {air }}\right) /\left(\epsilon_{\text {ice }}-\epsilon_{\text {air }}\right)-1\right)\right]$,

where $\epsilon_{\text {ice }}$ and $\epsilon_{\text {air }}$ are the dielectric constants of pure ice and air at $0^{\circ} \mathrm{C}$, and $f$ is the volume fraction of the tiny spheres. Because the planar ice crystals were modeled using three layers of tiny spheres, the volume fraction $f$ of the tiny spheres was set to $69 \%$ (i.e., the volume fraction of spheres closely packed into three layers infinite in extent), which is smaller than $74 \%$ because two-thirds of the spheres were at the surface. The resulting adjusted dielectric constant for the tiny spheres is listed in Table 1. After realizing the limitations of the GMM method in computing the single-scattering properties of pristine ice crystals, we performed DDA calculations for pristine ice crystals as a supplement.

The morphologies of the ice crystals used in the DDA calculations were generated in the same way as those used in the GMM calculations. Most of the planar ice crystals were modeled using 10 layers of dipoles, which was found to be a reasonable balance between accuracy and computational time, while ice crystals with aspect ratios close to unity were modeled using more layers of dipoles for accuracy because the overall number of dipoles used to model them was small. The dielectric constant of pure ice at $0^{\circ} \mathrm{C}$ was used in all DDA representations of a planar ice crystal.

\subsection{Columns}

The morphologies of columns were defined by their length $(L)$ and maximum dimension of their basal face $(d)$, constrained by the dimensional relationship

$d=3.0487 \times 10^{-2} L^{0.61078}$,

where $d$ and $L$ are in centimeters (Pruppacher and Klett, 1997, p. 51, Table 2.2b, N1a). Even though a needle model (N1a) was selected, thickness (maximum dimension of the basal face) variation factors of 1.0 and 2.0 were used to increase the range of variability of their properties. Therefore, we collectively call these crystals "columns" rather than "needles" because they are representative of the class of columnar crystals. In the GMM representation of ice columns, the columns have seven spheres along the diagonal (i.e., maximum dimension) of their basal faces. The ratio of the number of surface to interior tiny spheres was much lower than for planar crystals; hence their volume fractions were considered to be $74 \%$. The dielectric constant of the tiny spheres was once again adjusted so that the overall dielectric constant of the tiny sphere and air mixture composing the ice column was that of pure ice (Table 1). For the DDA calculations 16 dipoles were used along the diagonal of 


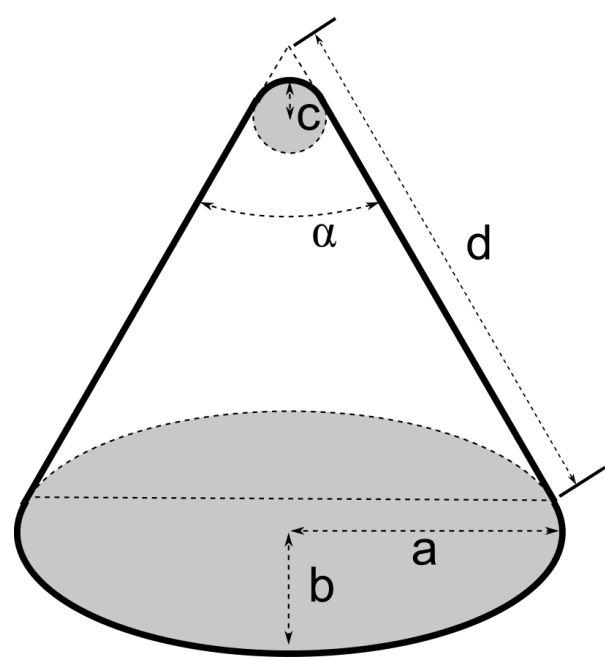

Figure 1. Two-dimensional slice through the axis of symmetry of a conical graupel particle, with the solid line representing the outline of the particle surface. The sphere with radius $c$, cone with apex angle $\alpha$ and height $d \cdot \cos (\alpha / 2)$, and spheroid with semi-major axis $a$ and semi-minor axis $b$ used to build the conical graupel particle are illustrated by shaded regions (within the particle) and dashed lines. The line labeled $d$ is tangent to both the sphere and spheroid. In this work, like in Aydin and Seliga (1984), we assume the constraints $b / a=0.5$ and $c / a=0.2$. The overall morphology of conical graupel in this representation is determined by its cone angle $\alpha$ and the spheroid semi-major axis $a$.

their basal faces. The dielectric constant of pure ice at $0{ }^{\circ} \mathrm{C}$ was used in all DDA representations of an ice column.

\subsection{Conical graupel}

The morphologies of conical graupel followed the spherecone-oblate-spheroid particle morphologies in Aydin and Seliga (1984), which are illustrated in their Fig. 1 and Fig. 1 here. The ratio of the semi-minor $(b)$ to semi-major $(a)$ axis of the spheroidal particle was set to $b / a=0.5$. The ratio of the radius $c$ of the sphere to the spheroidal semi-major axis was $c / a=0.2$. In this framework the shape of conical graupel was determined by its cone angle $\alpha$, and its size was determined by the value of $a$. Cone angles $\alpha$ ranged from 30 to $100^{\circ}$ in $10^{\circ}$ increments. For each cone angle, eight realizations of conical graupel were generated with equal-volumesphere radii of $0.2,0.3,0.4,0.5,1.0,1.5,2.0$, and $2.5 \mathrm{~mm}$. For each realization of a conical graupel particle 10 calculations were performed for particle densities of $0.05 \mathrm{~g} \mathrm{~cm}^{-3}$ and from 0.1 to $0.9 \mathrm{~g} \mathrm{~cm}^{-3}$ in $0.1 \mathrm{~g} \mathrm{~cm}^{-3}$ steps.

Because the DDA method has distinct advantages compared to GMM for computing the single-scattering properties of compact ice particles, only the DDA method was used to perform the calculations for conical graupel. The dielectric constants of the conical graupel were calculated using the Maxwell-Garnett formula (Bohren and Battan, 1980) with ice as the inclusion and air as the matrix. Substituting the pure ice dielectric constants at $0{ }^{\circ} \mathrm{C}$ and the ice volume fractions calculated based on the conical graupel densities, effective dielectric constants were calculated and used in the DDA calculations.

\subsection{Summary of ice-particle types in the database}

Table 2 contains a summary of the important parameters that differentiate ice particles within the aggregate, branched planar, plate, column, and conical graupel types. And Fig. 2 provides an illustration of a single ice particle within each type. All of this information is available within the Network Common Data Form (NetCDF) files that compose the database.

\section{Scattering geometry and reference frame}

Polarimetric radar observables are of value in ice-species classifications and ice water content retrievals. In order to retain polarimetric information for each ice-particle scattering calculation, we did not assume random orientations for the ice particles as doing so leads to the loss of some polarimetric information. Instead, the ice particles were fixed in orientation, and their single-scattering properties calculated for different directions of the incident radiation. For these calculations planar ice crystals were oriented with their basal faces parallel to the $x y$ plane and their maximum dimensions along the $x$ axis (Fig. 2). Ice columns had their lengths parallel to the $x$ axis as well, with the diagonals (maximum dimensions) of the basal faces aligned parallel to the $x y$ plane. For ice aggregates the axis of symmetry of the circumscribing reference spheroid was parallel to the $z$ axis, as was the axis of symmetry for conical graupel. We used one right-handed $x y z$ coordinate system, called the particle coordinate system, to describe the orientations of the ice particles. A second right-handed coordinate system was used to describe the direction of the incident radiation and the scattering plane. The orientation of this second coordinate system was obtained by first assuming it to be identical to the particle coordinate system. Then, the $x y$ plane of this second coordinate system was rotated by $\phi_{\mathrm{P}}^{\text {inc }}$ about the $z$ axis, with positive rotations representing a counterclockwise rotation when viewed in the negative $z$ axis direction (Fig. 3a). The newly oriented coordinate system, labeled with primes, was subsequently rotated by $\theta_{\mathrm{P}}^{\text {inc }}$ about the $y^{\prime}$ axis, to form the coordinate system labeled with double primes, so that the $z^{\prime \prime}$ axis of this coordinate system pointed in the direction of the incident radiation. For any direction of the scattered radiation the plane containing the incident and scattered radiation formed the scattering plane (Fig. 3b). The angle between the directions of the incident and scattered radiation (i.e., the scattering angle) was $\theta^{\text {sca }}$, and the angle between the projection of the scattered radiation onto the $x^{\prime \prime} y^{\prime \prime}$ plane and the $x^{\prime \prime}$ axis was $\phi^{\text {sca }}$ (Fig. 3b). 

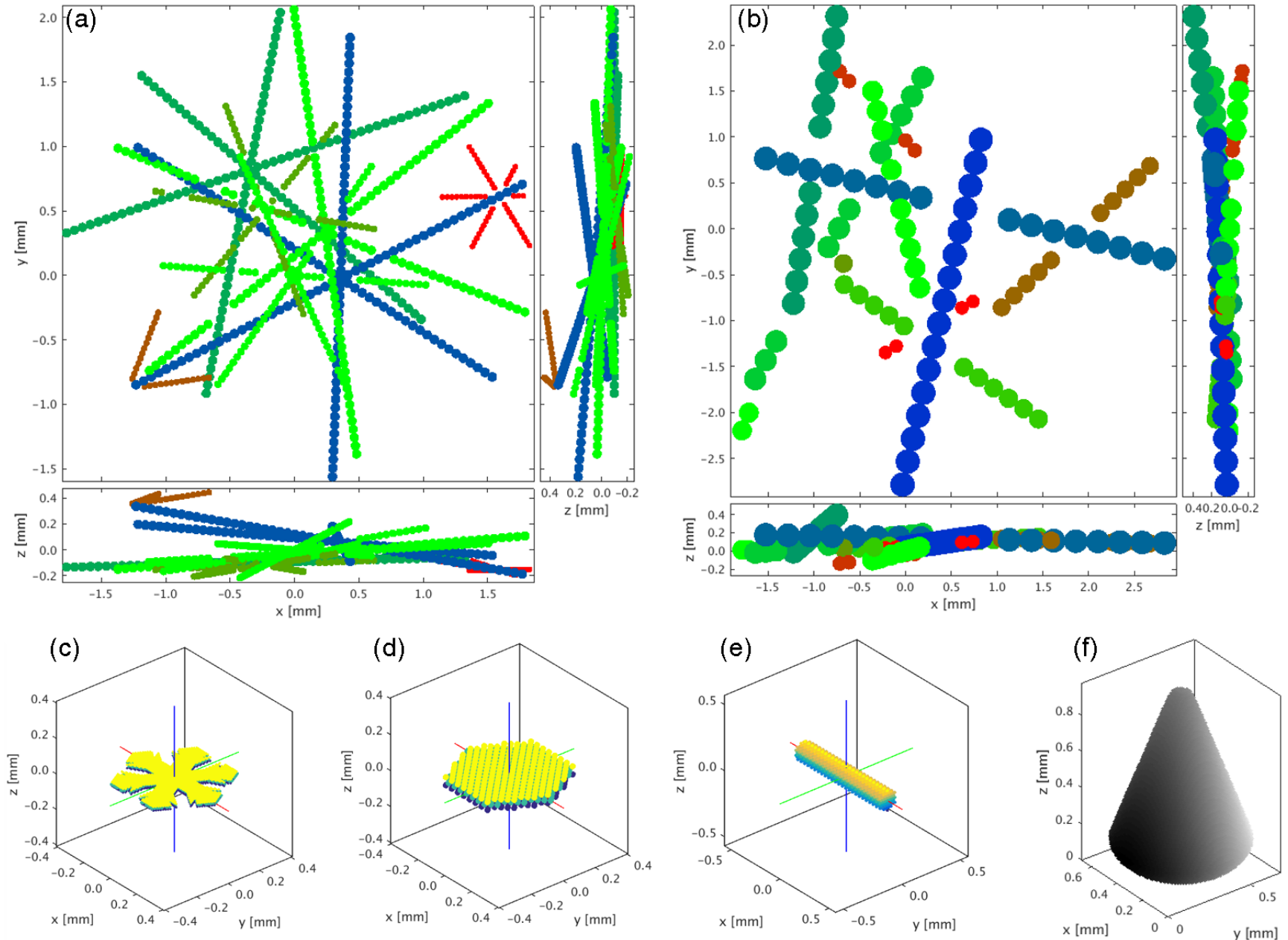

Figure 2. Examples of (a) an aggregate composed of stellar (color-coded) monomers, (b) an aggregate composed of column (color-coded) monomers, (c) a branched planar crystal, (d) a plate, (e) a column, and (f) a conical graupel particle. In panels (a)-(e) the representations are for GMM calculations, and the tiny spheres composing the representations are discernible. The conical graupel particle in (f) is represented as a field of indiscernible polarizable points on a rectangular lattice. Colors are used in (c)-(e) to distinguish different layers of the tiny spheres that compose the ice particles. Note that aggregate scattering properties are computed using GMM only, whereas graupel particle scattering properties are computed using DDA only; all other particle scattering properties are computed with both methods.

Scattering properties associated with different incident angles were calculated. The choice of the ranges and intervals of $\theta_{\mathrm{P}}^{\text {inc }}$ and $\phi_{\mathrm{P}}^{\text {inc }}$ were based on the symmetry properties of the ice particles. The aggregates had no symmetry axis, so $\theta_{\mathrm{P}}^{\text {inc }}$ varied from 0 to $180^{\circ}$, inclusive, in $10^{\circ}$ increments, while $\phi_{\mathrm{P}}^{\text {inc }}$ varied from 0 to $340^{\circ}$, inclusive, in $20^{\circ}$ increments. The ice crystal plates and branched planar crystals had a six-fold symmetry about their symmetry axis perpendicular to their basal faces, so $\theta_{\mathrm{P}}^{\text {inc }}$ varied from 0 to $90^{\circ}$, inclusive, in $10^{\circ}$ increments, while $\phi_{\mathrm{P}}^{\mathrm{inc}}$ was set to 0 and $30^{\circ}$. The conical graupel particles had rotational symmetry about their symmetry axis, so $\theta_{\mathrm{P}}^{\text {inc }}$ varied from 0 to $180^{\circ}$, inclusive, in $10^{\circ}$ increments, while $\phi_{\mathrm{P}}^{\text {inc }}$ equaled $0^{\circ}$. The ice columns had reflection symmetry, so $\theta_{\mathrm{P}}^{\text {inc }}$ varied from 0 to $90^{\circ}$, inclusive, in $10^{\circ}$ increments, while $\phi_{\mathrm{P}}^{\mathrm{inc}}$ also varied from 0 to $90^{\circ}$, inclusive, in $10^{\circ}$ increments. The orientation of the diagonals of the basal faces of ice columns had only a small influence on the singlescattering properties at the wavelengths investigated, so rotations of the basal face diagonals outside of the $x y$ plane were not considered.

For some of the ice-particle sizes and radiation wavelengths used to construct the database, lack of accurate interpolation from incident radiation directions in the database to new directions of incident radiation is by far its major limitation. This is true because some ice-particle single-scattering properties changed by significant amounts, and nonlinearly, over the angular intervals in $\theta_{\mathrm{P}}^{\mathrm{inc}}$ and $\phi_{\mathrm{P}}^{\mathrm{inc}}$ used to construct the database. Furthermore, this makes averaging over canting angle problematic for these particular ice particles. One possible means to accelerate the calculation of orientationaveraged single-scattering properties over a range of ice- 

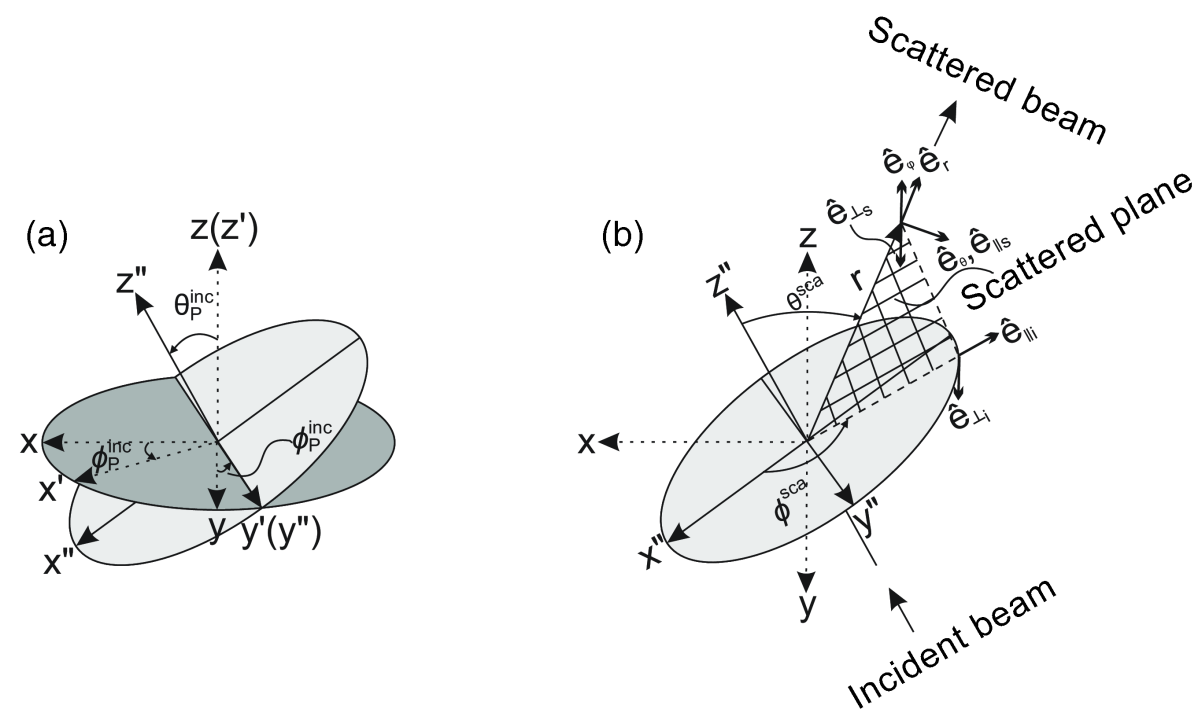

Figure 3. (a) The rotation angles, $\phi_{\mathrm{P}}^{\text {inc }}$ and $\theta_{\mathrm{P}}^{\text {inc }}$, necessary to orient the scattering coordinate system $\left(x^{\prime \prime} y^{\prime \prime} z^{\prime \prime}\right)$ relative to the particle coordinate system $(x y z)$. (b) Orientation of the scattered radiation, and hence the scattering plane, in the scattering coordinate system.

particle orientations is use of the invariant embedding (Johnson, 1988; Bi and Yang, 2014) or superposition (Mackowski and Mishchenko, 1996; Mackowski, 2014) T-matrix methods. In these approaches the T-matrix for an ice particle can be computed once and then used to calculate efficiently the single-scattering properties averaged over a range of iceparticle orientations.

\section{Ice-particle single-scattering properties in the database}

In the far field, the amplitude scattering matrix can be used to describe the relationship between the incident and scattered electric fields. Following the convention in Bohren and Huffman (1983, Sect. 3.2),

$\left[\begin{array}{c}E_{\| \mathrm{s}} \\ E_{\perp \mathrm{s}}\end{array}\right]=\frac{\exp (j k R)}{-j k R}\left[\begin{array}{ll}S_{2}\left(\theta^{\mathrm{sca}}, \phi^{\mathrm{sca}}\right) & S_{3}\left(\theta^{\mathrm{sca}}, \phi^{\mathrm{sca}}\right) \\ S_{4}\left(\theta^{\mathrm{sca}}, \phi^{\mathrm{sca}}\right) & S_{1}\left(\theta^{\text {sca }}, \phi^{\mathrm{sca}}\right)\end{array}\right]\left[\begin{array}{c}E_{\| \mathrm{i}} \\ E_{\perp \mathrm{i}}\end{array}\right]$

where $E$ is the electric field; the subscripts $\|$ and $\perp$ indicate parallel and perpendicular to the scattering plane (Fig. 3b); the subscripts $\mathrm{s}$ and $\mathrm{i}$ represent the scattered and incident radiation; $j$ is the square root of -1 ; and $k=2 \pi / \lambda$ is the wave number, where $\lambda$ is the wavelength of the radiation, $R$ is the distance between the ice particle and an observation point in the far field, and $S_{n}(n=1,2,3$, or 4$)$ are the elements of the amplitude scattering matrix, which are dimensionless under this convention and a function of the scattering polar angle $\theta^{\text {sca }}$ and scattering azimuth angle $\phi^{\text {sca }}$. For each triplet of a realization of an ice particle, radiation frequency, and incident radiation direction, the amplitude scattering matrices are computed and stored for every $1^{\circ}$ in $\theta^{\text {sca }}$ from 0 to $180^{\circ}$, inclusive, and every $5^{\circ}$ in $\phi^{\text {sca }}$ from 0 to $355^{\circ}$, inclusive.
Note that in the GMM output the amplitude scattering matrix is actually in terms of $S_{n}^{\prime}$ given by

$$
\left[\begin{array}{c}
E_{\| \mathrm{s}} \\
E_{\perp \mathrm{s}}
\end{array}\right]=\frac{\exp (j k R)}{-j k R}\left[\begin{array}{cc}
S_{2}^{\prime} & S_{3}^{\prime} \\
S_{4}^{\prime} & S_{1}^{\prime}
\end{array}\right]\left[\begin{array}{c}
E_{\| \mathrm{i}}^{\prime} \\
E_{\perp \mathrm{i}}^{\prime}
\end{array}\right],
$$

where $E_{\| i}^{\prime}$ and $E_{\perp i}^{\prime}$ are defined with respect to the $x^{\prime \prime} z^{\prime \prime}$ plane in Fig. $3 \mathrm{~b}$ rather than the scattering plane, while $E_{\| s}$ and $E_{\perp s}$ are still defined with respect to the scattering plane. The amplitude scattering matrix elements $S_{n}^{\prime}$ of the GMM output can be converted to the convention of Bohren and Huffman (1983) via the transformation

$$
\left[\begin{array}{ll}
S_{2} & S_{3} \\
S_{4} & S_{1}
\end{array}\right]=\left[\begin{array}{ll}
S_{2}^{\prime} & S_{3}^{\prime} \\
S_{4}^{\prime} & S_{1}^{\prime}
\end{array}\right]\left[\begin{array}{cc}
\cos \left(\phi^{\mathrm{sca}}\right) & \sin \left(\phi^{\mathrm{sca}}\right) \\
-\sin \left(\phi^{\mathrm{sca}}\right) & \cos \left(\phi^{\mathrm{sca}}\right)
\end{array}\right]
$$

The amplitude scattering matrix elements in the DDA output are of the same convention as Bohren and Huffman (1983). In construction of the database we adopted the convention of Bohren and Huffman (1983). The GMM output amplitude scattering matrices are first transformed to the convention of Bohren and Huffman (1983) before insertion into the database.

Once the amplitude scattering matrices are computed, all of the single-scattering properties follow. Because many of these single-scattering properties are frequently used in applications, they are reported in the database along with the amplitude scattering matrices. If a single-scattering property was not provided directly by the GMM or DDA codes, it was computed directly from the amplitude scattering matrices.

In radar meteorology the electric fields are usually broken down into components horizontal to the surface and perpendicular (vertical) to the horizontal component. With this geometry the amplitude scattering matrix in the forward- 
scatter alignment (FSA) convention of Bringi and Chandrasekar (2001) is defined as

$$
\left[\begin{array}{c}
E_{\mathrm{h}}^{\mathrm{s}} \\
E_{\mathrm{v}}^{\mathrm{s}}
\end{array}\right]=\frac{\exp (j k R)}{R}\left[\begin{array}{ll}
S_{\mathrm{hh}} & S_{\mathrm{hv}} \\
S_{\mathrm{vh}} & S_{\mathrm{vv}}
\end{array}\right]\left[\begin{array}{c}
E_{\mathrm{h}}^{\mathrm{i}} \\
E_{\mathrm{v}}^{\mathrm{i}}
\end{array}\right],
$$

where the subscripts $\mathrm{h}$ and $\mathrm{v}$ indicate horizontal and vertical polarizations, and the superscripts $s$ and $i$ represent the scattered and incident radiation. For the backward and forward scattering directions the scattering plane is not uniquely defined. For these two directions we adopt the convention of Bohren and Huffman (1983) in which the scattering plane rotates with $\phi^{\text {sca }}$. The scattering plane set by $\phi^{\text {sca }}=90^{\circ}$ is the $y^{\prime \prime} z^{\prime \prime}$ plane in Fig. 3b. In this case $\widehat{e}_{\|}$is always parallel to the horizontal polarization direction, and $\widehat{e}_{\perp}$ is always parallel to the vertical polarization direction no matter what the direction of the incident radiation. Therefore, if $\widehat{h}$ and $\widehat{v}$ represent horizontal and vertical polarization directions, then the wave propagation direction is $\widehat{v} \times \widehat{h}$, the same as $\widehat{e} \perp \times \widehat{e_{\|}}$.

The $S_{\mathrm{hh}}$ and $S_{\mathrm{vv}}$ amplitude scattering matrix elements for backward and forward scattering can be obtained from the dimensionless amplitude scattering matrix elements $S_{n}$ as

$$
S_{\mathrm{hh}}^{\mathrm{b}}=\frac{1}{-j k} S_{2}\left(180^{\circ}, 90^{\circ}\right), S_{\mathrm{vv}}^{\mathrm{b}}=\frac{1}{-j k} S_{1}\left(180^{\circ}, 90^{\circ}\right)
$$

(backward scattering);

$S_{\mathrm{hh}}^{\mathrm{f}}=\frac{1}{-j k} S_{2}\left(0^{\circ}, 90^{\circ}\right), S_{\mathrm{vv}}^{\mathrm{f}}=\frac{1}{-j k} S_{1}\left(0^{\circ}, 90^{\circ}\right)$

(forward scattering).

So the backscattering cross sections were computed as

$\sigma_{\mathrm{hh}}=4 \pi\left|S_{\mathrm{hh}}^{\mathrm{b}}\right|^{2}, \sigma_{\mathrm{vv}}=4 \pi\left|S_{\mathrm{vv}}^{\mathrm{b}}\right|^{2}$.

And $K_{\mathrm{DP}}$ produced by some concentration $n$ of a specific ice crystal can be computed as

$$
K_{\mathrm{DP}}=10^{3} \frac{180}{\pi} \frac{2 \pi}{k} n \operatorname{Re}\left(S_{\mathrm{hh}}^{\mathrm{f}}-S_{\mathrm{vv}}^{\mathrm{f}}\right)\left[^{\circ} \mathrm{km}^{-1}\right]
$$

\section{Structure of the database}

The database is stored in multiple NetCDF-formatted files for ease of use. The name of each file contains an identifier indicating whether the GMM or DDA method was used to produce the scattering properties that it contains. Each file contains the scattering properties associated with one individual ice particle at one frequency but for all directions of the incident and scattered radiation. Because there are 181 scattering (polar) angles (0 to $180^{\circ}$, inclusive, in $1^{\circ}$ increments) and 72 scattering azimuth angles (0 to $355^{\circ}$, inclusive, in $5^{\circ}$ increments) associated with the scattered radiation for each direction of the incident radiation, there are $181 \times 72$, i.e., 13032 , sets of amplitude scattering matrices associated with each direction of incident radiation. Because aggregates have the greatest number of directions of the incident radiation at 342 , they lead to the largest files, containing $342 \times 13032$ amplitude scattering matrices. Singlescattering properties that have only one value for each calculation (e.g., extinction cross section, absorption cross section, scattering cross section and backscattering cross section) or are of particular interest (e.g., forward-scattered differential phase) are extracted from each file and concatenated in a separate, much smaller, file. This smaller file is indexed via the following parameters: particle index indicating which particle is used in the calculation (e.g., 1 through 405 for branched planar crystals); frequency or wavelength indicators of the incident radiation (i.e., $\mathrm{W}-$, $\mathrm{Ka}-, \mathrm{Ku}-$, and $\mathrm{X}$-bands for the calculations currently in the database); polar angle $\theta_{\mathrm{P}}^{\text {inc }}$ of the incident radiation (e.g., 0 to $90^{\circ}$, inclusive, in $10^{\circ}$ increments for branched planar crystals); and azimuth angle $\phi_{\mathrm{P}}^{\mathrm{inc}}$ of the incident radiation (e.g., 0 and $30^{\circ}$ for branched planar crystals).

Physical properties related to each ice-particle realization are stored in this smaller file as variables with the particle index as the only dimension. These properties include ice-particle maximum dimension, thickness, mass, and projected area onto the $x y$ (horizontal) plane of the particle coordinate system. Detailed information of the ice particles in the database, including the location and radius of each tiny sphere used in the GMM representation of an ice particle or the locations of the polarizable points in the DDA representation of an ice particle, is provided in separate files for interested users. Finally, images of the ice particles in the database are also available.

The NetCDF files that compose the database are available through doi:10.5439/1258029 (Aydin et al., 2016). The dimensions, coordinate variables, geophysical variables, and file naming conventions for the NetCDF files are summarized in Tables S1-S5.

\section{Some illuminative results from the database}

The backscattering cross sections of the ice particles in the database are presented a number of different ways in Fig. 4. Focusing on the aggregates in the database (all of whose scattering properties were computed with the GMM), they range in mass from approximately $1 \times 10^{-3}$ to $20 \mathrm{mg}$ with backscattering cross sections at W-band from approximately $10^{-6}$ to $10^{1} \mathrm{~mm}^{2}$ (Fig. $4 \mathrm{~b}_{3}$ ). The aggregates in Leinonen and Moisseev (2015) range in mass from approximately $5 \times 10^{-3} \mathrm{mg}$ to just over $10 \mathrm{mg}$ with W-band backscattering cross sections from just below $1 \times 10^{-4} \mathrm{~mm}^{2}$ to just above $2 \times 10^{0} \mathrm{~mm}^{2}$ (their Fig. 2). While these ranges are comparable to each other, there are differences within them. For example, the drop in backscattering cross section that takes place when particle dimensions along the direction of the radiation reach about one-third of the wavelength (Fig. $\left.4 b_{1}, b_{2}\right)$ occurs at smaller particle masses here than in Leinonen and 

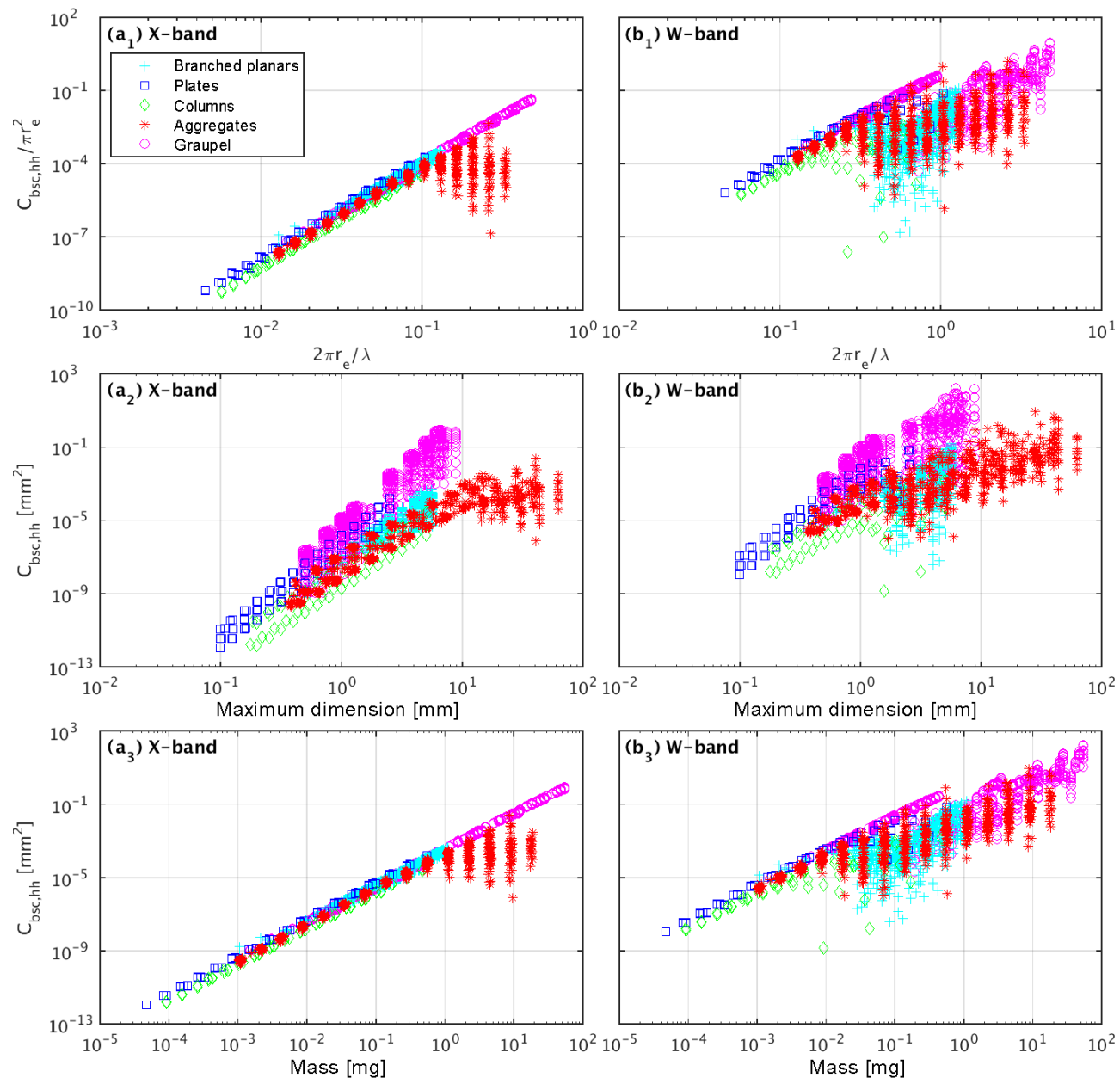

Figure 4. Horizontally (h)-polarized backscattering cross sections $C_{\mathrm{bsc}}$ hh for all ice particles in the database for side incident $\left(\theta_{\mathrm{P}}^{\text {inc }}=90^{\circ}\right.$, $\phi_{\mathrm{P}}^{\text {inc }}=0^{\circ}$ ) h-polarized radiation at (left column) X-band and (right column) W-band wavelengths. The backscattering cross sections are plotted vs. $\left(\mathbf{a}_{1}, \mathbf{b}_{1}\right)$ size parameter, $\left(\mathbf{a}_{2}, \mathbf{b}_{2}\right)$ maximum dimension, and $\left(\mathbf{a}_{3}, \mathbf{b}_{3}\right)$ mass. In $\left(\mathbf{a}_{1}, \mathbf{b}_{1}\right)$ each backscattering cross section is normalized by the cross sectional area of a solid $\left(0.917 \mathrm{~g} \mathrm{~cm}^{-3}\right)$ ice sphere with mass equal to that of the ice particle; $r_{\mathrm{e}}$ represents the radius of this equal-mass solid ice sphere.

Moisseev (2015). We attribute these differences between the two databases to the methods for building the aggregates and differences in the orientations of the aggregates for the backscattering calculations. The spacing in mass between ice particles is much smaller in Leinonen and Moisseev (2015) as they needed small steps in mass for their integrals of backscattering cross sections over aggregate size distributions.

Upon inspection of the X-band backscattering cross sections in the database, only the aggregates have maximum dimensions exceeding one-third the wavelength (Fig. 4a 2 ) and hence exhibit the drop in cross section with increasing size. For particles that are small compared to the wavelength, backscattering cross sections generally increase with mass (Fig. 4a $\mathrm{a}_{3}$ ) and its proxy $r_{\mathrm{e}}$ (Fig. 4a 1 ). The residual spread about the best-fit line to the backscattering cross sections at X-band wavelengths for ice-particle masses less than $1 \mathrm{mg}$, with the plates falling slightly above the line and columns slightly below the line, is explained by near-field interactions within the crystals ( $\mathrm{Lu}$ et al., 2013). The ratio of plate to column backscattering cross sections at $0.5 \mathrm{mg}$ is about 4 , or $6 \mathrm{~dB}$, which is quite close to the spread illustrated in Lu et al. (2013) for electric fields parallel and perpendicular to the basal faces of dendritic crystals.

Finally, the spread in the backscattering cross sections of ice particles at X-band wavelengths and at W-band wavelengths for side incident radiation of each ice-particle type for a given mass (Fig. $4 a_{3}, b_{3}$ ) is due to variations in morphologies for aggregate and branched planar particles, in thickness and maximum dimension for plate and column particles, and in cone angle and density for conical graupel. For branched planar, plate, and column particles, the different numerical methods also contribute to the spread. 

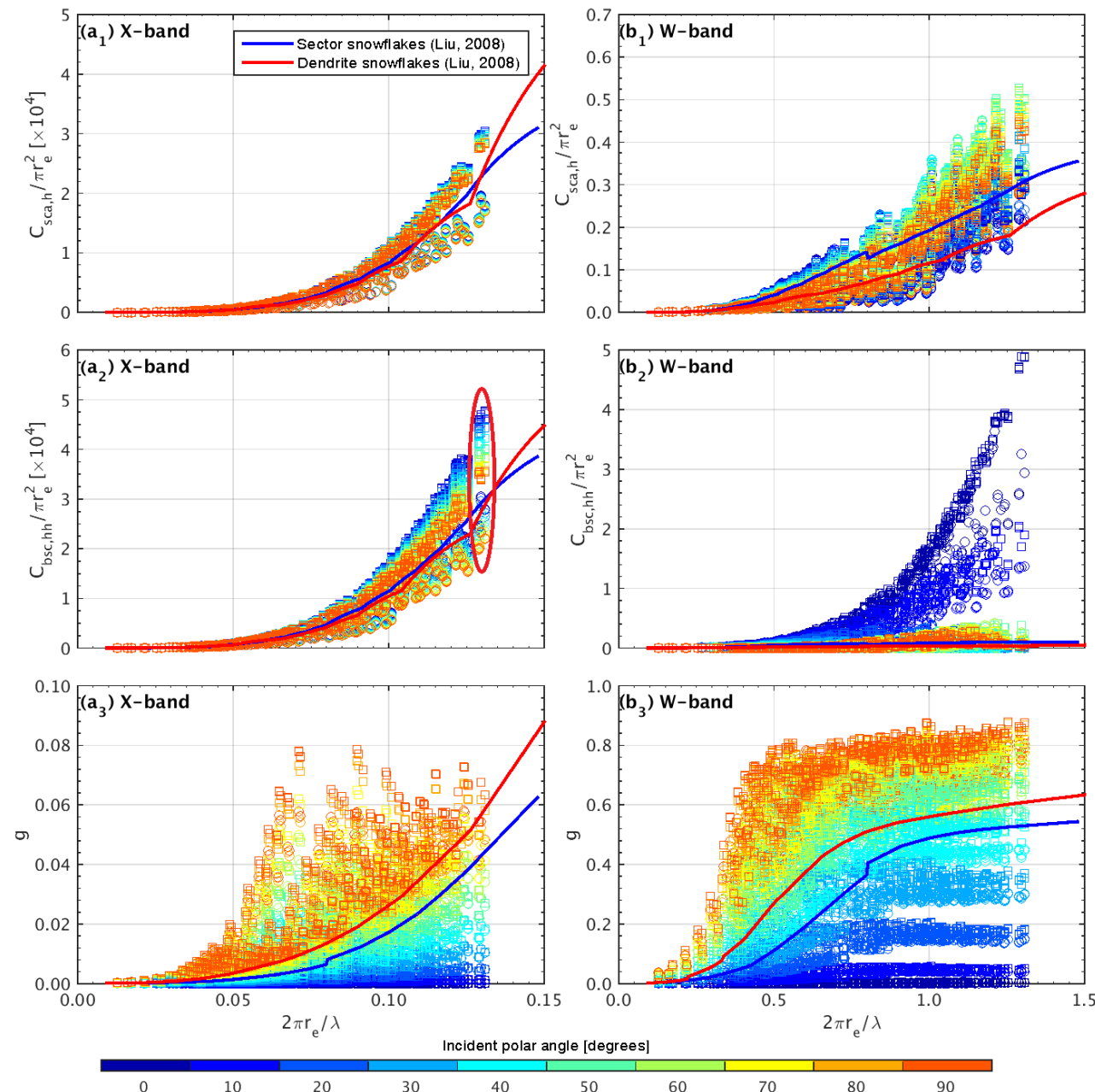

Figure 5. Database branched planar crystal $\left(\mathbf{a}_{1}, \mathbf{b}_{1}\right)$ scattering cross sections, $\left(\mathbf{a}_{2}, \mathbf{b}_{2}\right)$ backscattering cross sections, and $\left(\mathbf{a}_{3}, \mathbf{b}_{3}\right)$ asymmetry parameters for incident h-polarized radiation vs. size parameter $2 \pi r_{e} / \lambda$, where $\lambda$ is the wavelength of the incident radiation and $r_{\mathrm{e}}$ is the radius of a solid $\left(0.917 \mathrm{~g} \mathrm{~cm}^{-3}\right)$ ice sphere with mass equal to that of the crystal. GMM results are represented by open circles, whereas DDA results are given by open squares. The solid lines represent the results for (blue) sector snowflakes and (red) dendrite snowflakes from Liu (2008).

The database developed by Liu (2008) and Nowell et al. (2013) is well documented and openly accessible. In Fig. 5 scattering cross sections, backscattering cross sections, and asymmetry parameters computed via both DDA and GMM for branched planar crystals in the current database are compared to those of snowflakes in Liu (2008). Because Liu (2008) provides values averaged over random orientations of each snowflake and has a single snowflake for each maximum dimension (or mass represented by a single value of $r_{\mathrm{e}}$, where $r_{\mathrm{e}}$ is the radius of a solid $\left(0.916 \mathrm{~g} \mathrm{~cm}^{-3}\right)$ ice sphere with mass equal to that of the crystal), values for the two snowflake types in Liu (2008) vs. size parameter $2 \pi r_{\mathrm{e}} / \lambda$, where $\lambda$ is the wavelength of the incident radiation, can be represented as lines. For the current database the spread in values for fixed $r_{e}$ results from changes in ice-particle orientation with respect to the incident radiation, changes in ice-particle morphology, and differences between the DDA and GMM scattering methods.

For branched planar crystals oriented such that the normal to their basal planes is parallel to the incident radiation $\left(\theta_{\mathrm{P}}^{\text {inc }}=0^{\circ}\right)$, incident radiation scattered across the crystal constructively interferes in both the forward and backward scattering directions, because the thickness of each crystal is much smaller than the wavelength, resulting in induced dipoles that are all in phase. This, together with symmetry in the phases of the waves scattered into the forward and backward hemispheres, leads to asymmetry parameters that remain 0 for all crystal sizes (Fig. $5 \mathrm{a}_{3}, \mathrm{~b}_{3}$, dark blue circles). At oblique incident radiation, there are both constructive and destructive interferences for the scattered waves. As $\theta_{\mathrm{P}}^{\text {inc }}$ increases towards $90^{\circ}$, scattered waves in the forward direction retain their constructive interference, whereas waves scat- 

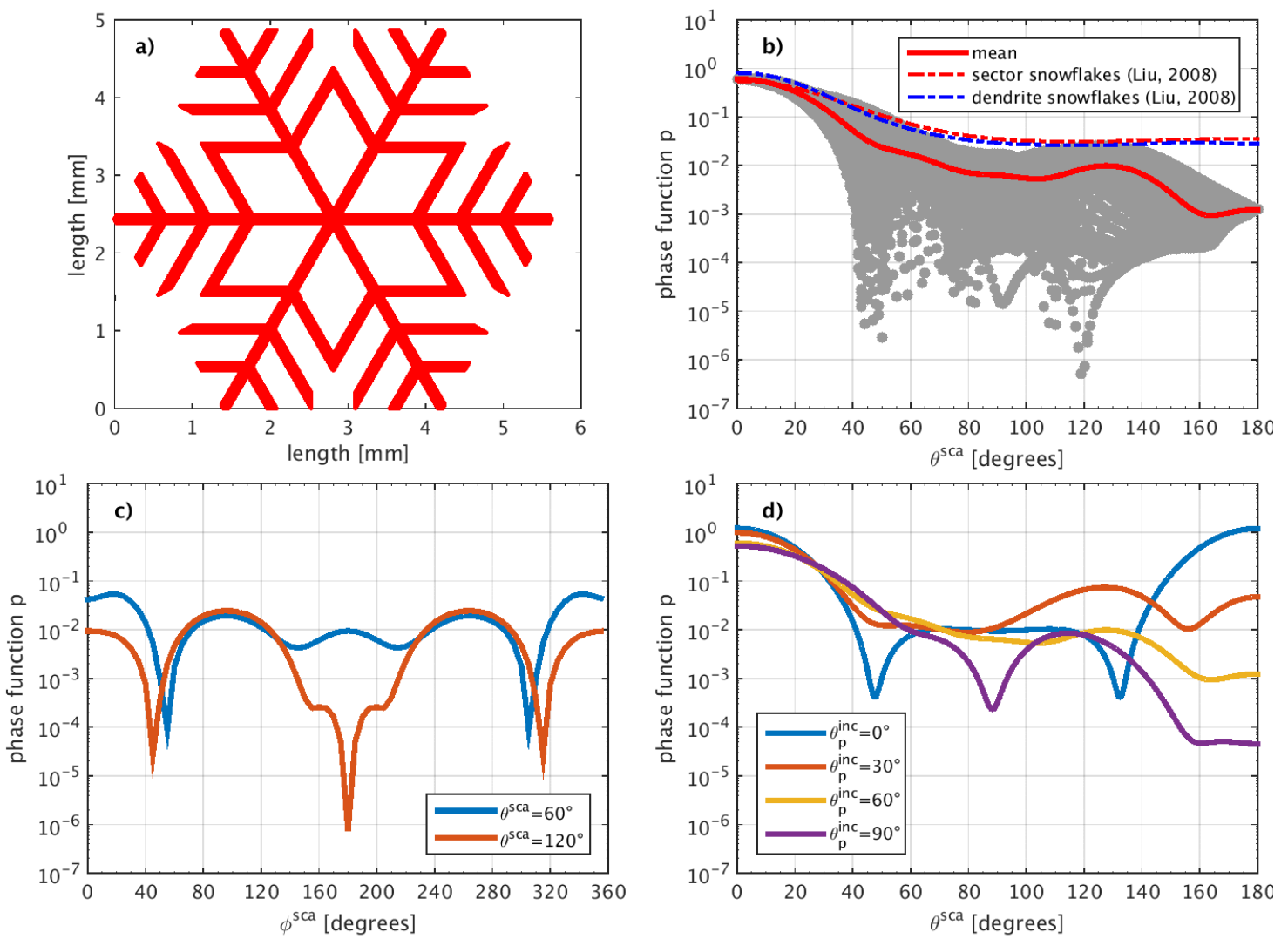

Figure 6. (a) Morphology of the 361 st branched planar crystal in the database. (b) The phase function $p$ (grey dots) of the 361 st branched planar crystal as a function of scattering polar angle $\theta^{\text {sca }}$ for incident radiation with $\theta_{\mathrm{P}}^{\text {inc }}=60^{\circ}$ and $\phi_{\mathrm{P}}^{\text {inc }}=0^{\circ}$; for each scattering polar angle $\theta^{\text {sca }}$ there are 72 grey dots, representing values for each of the 72 different scattering azimuth angles $\phi^{\text {sca }}$. The phase function averaged over the 72 values of $\phi^{\mathrm{sca}}$ is represented by the solid red line. The dashed red and blue lines represent the phase functions of the sector snowflake and dendrite snowflake from Liu (2008) with a similar maximum dimension. (c) The phase function $p$ of the 361 st branched planar crystal for (blue) $\theta^{\text {sca }}=60^{\circ}$ and (red) $\theta^{\text {sca }}=120^{\circ}$ as a function of scattering azimuth angle $\phi^{\text {sca }}$ for incident radiation with $\theta_{\mathrm{P}}^{\text {inc }}=60^{\circ}$ and $\phi_{\mathrm{P}}^{\text {inc }}=0^{\circ}$. (d) The phase functions $p$ (averaged over scattering azimuth angle $\phi^{\text {sca }}$ ) of the 361 st branched planar crystal as a function of scattering polar angle $\theta^{\text {sca }}$ for radiation with incident polar angles $\theta_{\mathrm{P}}^{\text {inc }}=0,30,60$, and $90^{\circ}$ and with incident azimuth angle $\phi_{\mathrm{P}}^{\text {inc }}=0^{\circ}$. The results in (b), (c), and (d) were obtained from DDA calculations at the W-band wavelength.

tered in other directions, including the backward direction, lose it. This is especially true at the smaller W-band wavelength as the planar crystals increase in maximum dimension. As this happens, forward scattering dominates, with asymmetry parameters of the largest planar crystals at the W-band wavelength reaching values near 0.9 at side incidence $\left(\theta_{\mathrm{P}}^{\text {inc }}=90^{\circ}\right.$; Fig. $5 \mathrm{~b}_{3}$, red circles $)$. Though these tendencies are also apparent at the $\mathrm{X}$-band wavelength, they are much smaller because all branched planar crystals in the database are small compared to (less than one-third of) the wavelength. As a result, at the X-band wavelength the asymmetry parameters never exceed 0.08 .

The loss in constructive interference in the backward scattering direction for increasing incident polar angle $\theta_{\mathrm{P}}^{\text {inc }}$ is also reflected in drops in the backscattering cross sections, once again with the drop increasing with branched planar crystal maximum dimension and with it being significantly greater at the W-band wavelength compared to the X-band wavelength (Fig. $5 \mathrm{a}_{2}, \mathrm{~b}_{2}$ ).

Carefully inspecting the backscattering cross sections at the X-band wavelength near a size parameter of 0.13 (red oval in Fig. 5a 2 ), one finds eight different particles contributing to them. As expected, the backscattering cross sections for all eight particles decrease with increasing incident polar angle. However, four of the particles have significantly larger backscattering cross sections at $\theta_{\mathrm{P}}^{\text {inc }}=0^{\circ}$ than the other four. These differences result from the method (i.e., DDA vs. GMM) used to compute the scattering properties. The four sets with the largest backscattering cross sections were computed with DDA, whereas the four sets with the smallest backscattering cross sections are for the same four branched planar crystals but computed via GMM. The backscattering 
cross sections in Fig. 5a that fall below those for the sector and dendrite snowflakes in Liu (2008) all result from GMM calculations, though many GMM calculations also lie above them. That the Liu (2008) backscattering cross sections split the difference between the DDA and some GMM results in the current database was not by design. All of these same features are evident in the scattering cross sections in Fig. 5a though the decrease with increasing incident polar angle $\theta_{\mathrm{P}}^{\text {inc }}$ is not as large because the scattering cross sections are an integral quantity that includes contributions from directions other than backward.

Differences in the scattering cross sections at the Wband wavelength between DDA and GMM are again evident (Fig. 5b 1 ). However, for size parameters larger than 0.75 the branched planar crystals are sufficiently large that complicated patterns of constructive and destructive interference vs. scattering angles $\left(\theta^{\text {sca }}, \phi^{\text {sca }}\right)$ take place for all incident polar angles $\theta_{\mathrm{P}}^{\text {inc }}$. As a result, variability is driven by the incident polar angle, and the scattering cross sections first increase and then decrease with incident polar angle, reaching maximum values near $\theta_{\mathrm{P}}^{\text {inc }}=50^{\circ}$.

Richness in the directionality of the scattering for branched planar crystals that are large compared to the $\mathrm{W}$ band wavelength is illustrated in Fig. 6. Figure 6a is a depiction of the branched planar crystal upon which the Fig. 6 results are based; it has a maximum dimension greater than $5.5 \mathrm{~mm}$ and is nearly twice the wavelength. For unpolarized radiation with an incident polar angle $\theta_{\mathrm{P}}^{\text {inc }}=60^{\circ}$ and incident azimuth angle $\phi_{\mathrm{P}}^{\text {inc }}=0^{\circ}$, the resulting scattering phase function lacks azimuthal symmetry except for scattering angles close to the forward and backward directions (Fig. 6b, grey dots; Fig. 6c, blue and red lines). This lack of azimuthal symmetry is one motivating factor for not reporting a scattering phase function in the database while retaining the amplitude scattering matrices at $1^{\circ}$ intervals in scattering polar angle and $5^{\circ}$ intervals in scattering azimuth angle. The scattering phase function for a specific direction can be calculated from the amplitude scattering matrices following Bohren and Huffman (1983, pp. 71-72). Averaging the scattering phase function over all azimuth angles for fixed scattering polar angle leads to smooth dependence on scattering polar angle (Fig. 6b, solid red line) with similar values in the forward scattering direction to the scattering phase functions for randomly oriented snowflakes (Fig. 6b, dashed red and blue lines; Liu, 2008).

As Liu (2008) points out, when computing the scattering properties of a randomly oriented ice particle, one must average over small changes in the orientation of an ice particle, especially in order to compute accurate backscattering cross sections. This being the case is evident in Fig. 6d, where the scattering phase function does not depend strongly on the orientation of the branched planar crystal for forward directions, where constructive interference always occurs. In the backward direction the scattering phase function is highly dependent on the ice-particle orientation, dropping by 4 orders of magnitude as the incident polar angle changes from $\theta_{\mathrm{P}}^{\text {inc }}=0^{\circ}$, for which the asymmetry parameter is 0 , to $\theta_{\mathrm{P}}^{\text {inc }}=90^{\circ}$, for which the asymmetry parameter is much closer to 1 .

The imaginary part of the refractive index of ice is about 4 orders of magnitude smaller than its real part at radar wavelengths (Table 1) and is sensitive to the dielectric constant adjustment that we used to compensate for the tiny sphere and air-gap mixture representation of ice particles in the GMM scattering calculations. Moreover, the imaginary part can have large relative changes over temperatures ranging from -40 to $0^{\circ} \mathrm{C}$ (Mätzler, 2006). Because the current database incorporated dielectric constants from Ray (1972), the imaginary parts of which are significantly smaller than those of Mätzler (2006), and because we made adjustments to them for the GMM calculations that may have substantially altered their imaginary parts, the absorption cross sections in this database should be used with caution.

\section{Summary and future refinements}

A database of single-scattering properties of ice particles at millimeter to centimeter wavelengths is presented to facilitate ground-based, airborne, and satellite-based retrievals of ice cloud and ice precipitation properties. Branched planar crystals, plates, columns, aggregates, and conical graupel are generated, and their single-scattering properties calculated, using the GMM and/or DDA methods for different directions of the incident radiation. In addition to the scattering properties of each ice particle, including their amplitude scattering matrices as a function of incident and scattered directions, which provide full polarization information, the database also contains the physical properties of each ice particle, including the location of each ice-particle component (a tiny sphere in the GMM calculations and a polarizable point in the DDA calculations), together with imagery of it. The addition of new ice-particle realizations or new ice-particle species, such as rimed or melting particles, will be incorporated into the database as they become available and then subsequently documented.

There are several limitations to the current database. First, the ice particles in the database are just a small representation of the ice particles that exist in nature. Direct observations of exact morphologies of real ice particles, especially those in clouds, are limited. Improvements in the observation of detailed ice-particle structures would serve as valuable guidance in the future development of the database. Second, calculations at high frequencies for more directions of the incident radiation will be necessary to ensure accurate interpolation of ice-particle single-scattering properties to other directions of the incident radiation. And finally, the absorption cross sections in the database should be used with caution as they are based on older dielectric constants (Ray, 1972), 
and in the case of the GMM calculations may have errors in them as a result of adjustments made to compensate for the air gaps in the tiny sphere representations of the ice particles.

\section{Data availability}

The database is available through doi:10.5439/1258029 (Aydin et al., 2016).

\section{The Supplement related to this article is available online at doi:10.5194/amt-9-5119-2016-supplement.}

Acknowledgements. We would like to thank Giriprakash Palanisamy and Kathy Lazar for their invaluable help in setting up the database as a Department of Energy (DOE) Atmospheric Radiation Measurement (ARM) Program Principal Investigator Data Product that comes with a DOI. Raymond McCord and Kenneth Kehoe, also of the DOE ARM Program, provided many useful comments regarding the NetCDF4 files in which the database is housed. Discussions with Stefan Kneifel, Ralf Bennartz, Alan Geer, Pavlos Kollias, Ann Fridlind, Greg McFarquhar, Christopher Williams, and Stephen Nesbit were helpful in framing the scope of the database products and/or generating interest within the DOE ARM Program in hosting it. Development of the database was supported primarily by NSF Grant AGS-128180. Eugene E. Clothiaux's contributions to the database were supported by DOE Grant DE-FG02-05ER64058. The authors would like to acknowledge high-performance computing support from Yellowstone (ark:/85065/d7wd3xhc) provided by NCAR's Computational and Information Systems Laboratory, sponsored by the National Science Foundation. Portions of this research were conducted with Advanced Cyberinfrastructure computational resources provided by the Institute for CyberScience at The Pennsylvania State University (http://ics.psu.edu)

Edited by: M. Kulie

Reviewed by: two anonymous referees

\section{References}

Aydin, K. and Seliga, T. A.: Radar Polarimetric Backscattering Properties of Conical Graupel, J. Atmos. Sci., 41, 1887-1892, doi:10.1175/1520-0469(1984)041<1887:RPBPOC >2.0.CO;2, 1984.

Aydin, K. and Singh, J.: Cloud Ice Crystal Classification Using a $95 \mathrm{GHz}$ Polarimetric Radar, J. Atmos. Ocean. Tech., 21, 16791688, doi:10.1175/JTECH1671.1, 2004.

Aydin, K. and Tang, C.: Relationships between IWC and Polarimetric Radar Measurands at 94 and $220 \mathrm{GHz}$ for Hexagonal Columns and Plates, J. Atmos. Ocean. Tech., 14, 1055-1063, doi:10.1175/1520-0426(1997)014<1055:RBIAPR>2.0.CO;2, 1997.
Aydin, K., Verlinde, J., Clothiaux, E. E., Lu, Y., Jiang, Z., and Botta, G.: Polarimetric scattering database for non-spherical ice particles at microwave wavelengths. Atmospheric Radiation Measurement (ARM) Climate Research Facility Data Archive: Oak Ridge, Tennessee, USA, Data set available at: doi:10.5439/1258029, 2016.

Battaglia, A., Westbrook, C. D., Kneifel, S., Kollias, P., Humpage, N., Löhnert, U., Tyynelä, J., and Petty, G. W.: G band atmospheric radars: new frontiers in cloud physics, Atmos. Meas. Tech., 7, 1527-1546, doi:10.5194/amt-7-1527-2014, 2014.

Bi, L. and Yang, P.: Accurate simulation of the optical properties of atmospheric ice crystals with the invariant imbedding T-matrix method, J. Quant. Spectrosc. Ra., 138, 17-35, doi:10.1016/j.jqsrt.2014.01.013, 2014.

Bringi, V. N. and Chandrasekar, V.: Polarimetric Doppler weather radar: principles and applications, Cambridge University Press, 45-48, 2001.

Bohren, C. F. and Battan, L. J.: Radar Backscattering by Inhomogeneous Precipitation Particles, J. Atmos. Sci., 37, 1821-1827, doi:10.1175/1520-0469(1980)037<1821:RBBIPP>2.0.CO;2, 1980.

Bohren, C. F. and Huffman, D. R.: Absorption and scattering of light by small particles, 1st ed. Wiley, New York, 57-81, 1983.

Botta, G., Aydin, K., and Verlinde, J.: Modeling of microwave scattering from cloud ice crystal aggregates and melting aggregates: a new approach, IEEE Geosci. Remote S., 7, 572-576, doi:10.1109/LGRS.2010.2041633, 2010.

Botta, G., Aydin, K., Verlinde, J., Avramov, A. E., Ackerman, A. S., Fridlind, A., McFarquhar, G. M., and Wolde, M.: Millimeter wave scattering from ice crystals and their aggregates: Comparing cloud model simulations with $\mathrm{X}$ - and Kaband radar measurements, J. Geophys. Res., 116, D00T04, doi:10.1029/2011JD015909, 2011.

Botta, G., Aydin, K., and Verlinde, J.: Variability in millimeter wave scattering properties of dendritic ice crystals, J. Quant. Spectrosc. Ra., 131, 105-114, doi:10.1016/j.jqsrt.2013.05.009, 2013.

Chandrasekar, V., Keränen, R., Lim, S., and Moisseev, D.: Recent advances in classification of observations from dual polarization weather radars, Atmos. Res., 119, 97-111, doi:10.1016/j.atmosres.2011.08.014, 2013.

Computational and Information Systems Laboratory: Yellowstone: IBM iDataPlex System (University Community Computing), Boulder, CO: National Center for Atmospheric Research, available at: http://n2t.net/ark:/85065/d7wd3xhc (last access: August 2015), 2012.

Draine, B. T. and Flatau, P. J.: Discrete-dipole approximation for scattering calculations, J. Opt. Soc. Amer. A, 11, 1491, doi:10.1364/JOSAA.11.001491, 1994.

Galligani, V. S., Prigent, C., Defer, E., Jimenez, C., Eriksson, P., Pinty, J.-P., and Chaboureau, J.-P.: Meso-scale modelling and radiative transfer simulations of a snowfall event over France at microwaves for passive and active modes and evaluation with satellite observations, Atmos. Meas. Tech., 8, 1605-1616, doi:10.5194/amt-8-1605-2015, 2015.

Geer, A. J. and Baordo, F.: Improved scattering radiative transfer for frozen hydrometeors at microwave frequencies, Atmos. Meas. Tech., 7, 1839-1860, doi:10.5194/amt-7-1839-2014, 2014. 
Goodman, J. J., Draine, B. T., and Flatau, P. J.: Application of fastFourier-transform techniques to the discrete-dipole approximation, Opt. Lett., 16, 1198, doi:10.1364/OL.16.001198, 1991.

Hou, A. Y., Kakar, R. K., Neeck, S., Azarbarzin, A. A., Kummerow, C. D., Kojima, M., Oki, R., Nakamura, K., and Iguchi, T.: The Global Precipitation Measurement Mission, B. Am. Meteorol. Soc., 95, 701-722, doi:10.1175/BAMS-D-13-00164.1, 2014.

IPCC: Climate Change 2013: The Physical Science Basis, in: Contribution of Working Group I to the Fifth Assessment Report of the Intergovernmental Panel on Climate Change, edited by: Stocker, T. F., Qin, D., Plattner, G.-K., Tignor, M., Allen, S. K., Boschung, J., Nauels, A., Xia, Y., Bex, V. and Midgley, P. M., Cambridge University Press, Cambridge, United Kingdom and New York, NY, USA, 1535 pp., 2013.

Johnson, B. T., Petty, G. W., and Skofronick-Jackson, G.: Microwave properties of ice-phase hydrometeors for radar and radiometers: Sensitivity to model assumptions, J. Appl. Meteorol. Climatol., 51, 2152-2171, 2012.

Kim, M.-J.: Single scattering parameters of randomly oriented snow particles at microwave frequencies, J. Geophys. Res., 111, D14201, doi:10.1029/2005JD006892, 2006.

Kneifel, S., Kulie, M. S., and Bennartz, R.: A triple-frequency approach to retrieve microphysical snowfall parameters, J. Geophys. Res. Atmos., 116, D11203, doi:10.1029/2010JD015430, 2011

Kneifel, S., von Lerber, A., Tiira, J., Moisseev, D., Kollias, P., and Leinonen, J.: Observed relations between snowfall microphysics and triple-frequency radar measurements, J. Geophys. Res. Atmos., 120, 6034-6055, doi:10.1002/2015JD023156, 2015.

Kulie, M. S., Bennartz, R., Greenwald, T. J., Chen, Y., and Weng, F.: Uncertainties in microwave properties of frozen precipitation: Implications for remote sensing and data assimilation, J. Atmos. Sci., 67, 3471-3487, 2010.

Kulie, M. S., Hiley, M. J., and Bennartz, R.: Triple-frequency radar reflectivity signatures of snow: Observations and comparisons with theoretical ice particle scattering models, J. Appl. Meteorol. Clim., 53, 1080-1098, 2014.

Kumjian, M. R.: Principles and applications of dual-polarization weather radar. Part I: Description of the polarimetric radar variables, J. Operational Meteor., 1, 226-242, doi:10.15191/nwajom.2013.0119, 2013.

Kuo, K. S., Olson, W. S., Johnson, B. T., Grecu, M., Tian, L., Clune, T. L., van Aartsen, B. H., Heymsfield, A. J., Liao, L., and Meneghini, R.: The microwave radiative properties of falling snow derived from nonspherical ice particle models. part I: an extensive database of simulated pristine crystals and aggregate particles, and their scattering properties, J. Appl. Meteorol. Clim., 55, 691-708, 2016.

Leinonen, J. and Moisseev, D.: What do triple-frequency radar signatures reveal about aggregate snowflakes?, J. Geophys. Res.Atmos., 120, 229-239, doi:10.1002/2014JD022072, 2015.

Leinonen, J., Kneifel, S., Moisseev, D., Tyynelä, J., Tanelli, S., and Nousiainen, T.: Evidence of nonspheroidal behavior in millimeter-wavelength radar observations of snowfall, J. Geophys. Res.-Atmos., 117, D18205, doi:10.1029/2012JD017680, 2012.

Leinonen, J., Lebsock, M. D., Tanelli, S., Suzuki, K., Yashiro, H., and Miyamoto, Y.: Performance assessment of a triplefrequency spaceborne cloud-precipitation radar concept using a global cloud-resolving model, Atmos. Meas. Tech., 8, 34933517, doi:10.5194/amt-8-3493-2015, 2015.

Liu, C., Lee Panetta, R., and Yang, P.: Application of the pseudo-spectral time domain method to compute particle single-scattering properties for size parameters up to 200, J. Quant. Spectrosc. Ra., 113, 1728-1740, doi:10.1016/j.jqsrt.2012.04.021, 2012.

Liu, G.: A database of microwave single-scattering properties for nonspherical ice particles, B. Am. Meteorol. Soc., 89, 15631570, doi:10.1175/2008BAMS2486.1, 2008.

Lu, Y., Clothiaux, E. E., Aydin, K., Botta, G., and Verlinde, J.: Modeling variability in dendritic ice crystal backscattering cross sections at millimeter wavelengths using a modified Rayleigh-Gans theory, J. Quant. Spectrosc. Ra., 131, 95-104, doi:10.1016/j.jqsrt.2013.05.008, 2013.

Lu, Y., Aydin, K., Clothiaux, E. E., and Verlinde, J.: Dielectric constant adjustments in computations of the scattering properties of solid ice crystals using the Generalized Multiparticle Mie method, J. Quant. Spectrosc. Ra., 135, 1-8, doi:10.1016/j.jqsrt.2013.12.005, 2014a.

Lu, Y., Clothiaux, E. E., Aydin, K., and Verlinde, J.: Estimating ice particle scattering properties using a modified RayleighGans approximation, J. Geophys. Res., 119, 10471-10484, doi:10.1002/2014JD021850, 2014b.

Lu, Y., Aydin, K., Clothiaux, E. E., and Verlinde, J.: Retrieving Cloud Ice Water Content Using Millimeter- and CentimeterWavelength Radar Polarimetric Observables, J. Appl. Meteorol. Clim., 54, 596-604, doi:10.1175/JAMC-D-14-0169.1, 2015.

Mackowski, D. W.: A general superposition solution for electromagnetic scattering by multiple spherical domains of optically active media, J. Quant. Spectrosc. Ra., 133, 264-270, doi:10.1016/j.jqsrt.2013.08.012, 2014.

Mackowski, D. W. and Mishchenko, M. I.: Calculation of the T matrix and the scattering matrix for ensembles of spheres, J. Opt Soc. Amer. A, 13, 2266-2278, 1996.

Maruyama, K.-I. and Fujiyoshi, Y.: Monte carlo simulation of the formation of snowflakes, J. Atmos. Sci., 62, 1529-1544, 2005.

Matrosov, S. Y.: A dual-wavelength radar method to measure snowfall rate, J. Appl. Meteorol., 37, 1510-1521, 1998.

Mätzler, C.: Microwave dielectric properties of ice, in: Thermal microwave radiation-applications for remote sensing, Electromagnetic Waves Series, edited by: Mätzler C., vol. 52, Stevenage, UK, The Institution of Engineering and Technology, 455-462, 2006.

Mitchell, D. L.: Use of mass-and area-dimensional power laws for determining precipitation particle terminal velocities, J. Atmos. Sci., 53, 1710-1723, 1996.

Nowell, H., Liu, G., and Honeyager, R.: Modeling the microwave single-scattering properties of aggregate snowflakes, J. Geophys. Res., 118, 7873-7885, 2013.

Oue, M., Kumjian, M. R., Lu, Y., Jiang, Z., Clothiaux, E. E., Verlinde, J., and Aydin, K.: X-band polarimetric and Ka-band Doppler spectral radar observations of a graupel-producing Arctic mixed-phase cloud, J. Appl. Meteorol. Clim., 54, 1335-1351, doi:10.1175/JAMC-D-14-0315.1, 2015.

Petty, G. W. and Huang, W.: Microwave backscatter and extinction by soft ice spheres and complex snow aggregates, J. Atmos. Sci., 67, 769-787, doi:10.1175/2009JAS3146.1, 2010. 
Pruppacher, H. R. and Klett, J. D.: Microphysics of clouds and precipitation. 2nd rev. a. Kluwer Academic Publishers, Dordrecht, the Netherlands, 1997.

Purcell, E. M. and Pennypacker, C. R.: Scattering and absorption of light by nonspherical dielectric grains, Astrophys. J., 186, 705, doi:10.1086/152538, 1973.

Ray, P. S.: Broadband complex refractive indices of ice and water, Appl. Optics, 11, 1836-1844, 1972.

Ryzhkov, A. V., Zrnic, D. S., and Gordon, B. A.: Polarimetric Method for Ice Water Content Determination, J. Appl. Meteor., 37, 125-134, doi:10.1175/15200450(1998)037<0125:PMFIWC>2.0.CO;2, 1998.

Stein, T. H. M., Westbrook, C. D., and Nicol, J. C.: Fractal geometry of aggregate snowflakes revealed by triple-wavelength radar measurements, Geophys. Res. Lett., 42, 176-183, 2015.

Straka, J. M., Zrnić, D. S., and Ryzhkov, A. V.: Bulk hydrometeor classification and quantification using polarimetric radar data: synthesis of relations, J. Appl. Meteorol., 39, 1341-1372, 2000.

Takahashi, T., Endoh, T., Wakahama, G., and Fukuta, N.: Vapor Diffusional Growth of Free-Falling Snow Crystals between -3 and $-23^{\circ} \mathrm{C}$, J. Meteor. Soc. Japan, 69, 251-251, 1991.
Tavlove, A. and Hagness, S.: Computational electrodynamics: the finite-difference time-domain method, 3rd ed., Chapter 1, Artech House, Boston, 2005.

Tyynelä, J. and Chandrasekar, V.: Characterizing falling snow using multifrequency dual-polarization measurements, J. Geophys. Res.-Atmos., 119, 8268-8283, doi:10.1002/2013JD021369, 2014.

Westbrook, C. D., Ball, R. C., Field, P. R., and Heymsfield, A. J.: Universality in snowflake aggregation, Geophys. Res. Lett., 31, L15104, doi:10.1029/2004GL020363, 2004.

$\mathrm{Xu}$, Y.: Electromagnetic scattering by an aggregate of spheres, Appl. Opt., 34, 4573-4588, doi:10.1364/AO.34.004573, 1995.

Yee, K.: Numerical solution of initial boundary value problems involving maxwell's equations in isotropic media, IEEE Trans. Antennas Propag., 14, 302-307, doi:10.1109/TAP.1966.1138693, 1966.

Yurkin, M. A. and Hoekstra, A. G.: The discrete-dipoleapproximation code ADDA: Capabilities and known limitations, J. Quant. Spectrosc. Ra., 112, 2234-2247, doi:10.1016/j.jqsrt.2011.01.031, 2011. 